\title{
High-Frequency Analysis of Carbon Nanotube Interconnects and Implications for On-Chip Inductor Design
}

\author{
Hong Li, Student Member, IEEE, and Kaustav Banerjee, Senior Member, IEEE
}

\begin{abstract}
This paper presents a rigorous investigation of highfrequency effects in carbon nanotube (CNT) interconnects and their implications for the design and performance analysis of highquality on-chip inductors. A frequency-dependent impedance extraction method is developed for both single-walled CNT (SWCNT) and multiwalled CNT (MWCNT) bundle interconnects. The method is subsequently verified by comparing the results with those derived directly from the Maxwell's equations. Our analysis reveals for the first time that skin effect in CNT (particularly MWCNT) bundles is significantly reduced compared to that in conventional metal conductors, which makes them very attractive and promising material for high-frequency applications, including high-quality $(Q)$ factor on-chip inductor design in highperformance $\mathrm{RF} / \mathrm{mixed}$-signal circuits. It is shown that such unique high-frequency properties of CNTs essentially arise due to their large momentum relaxation time (leading to their large kinetic inductance), which causes the skin depths to saturate with frequency and thereby limits resistance increase at high frequencies in a bundle structure. It is subsequently shown that CNTbased planar spiral inductors can achieve more than three times higher $Q$ factor than their Cu-based counterparts without using any magnetic materials or $Q$ factor enhancement techniques.
\end{abstract}

Index Terms-AC conductivity, carbon nanotube (CNT), energy storage, high-frequency, interconnect, momentum relaxation time, on-chip inductor, $Q$ factor, skin depth, skin effect.

\section{INTRODUCTION}

C ARBON nanotubes (CNTs) have been proposed for VLSI interconnect applications [1] due to their outstanding electrical and thermal properties [2]-[5]. Several works have investigated the circuit performance of CNT interconnects. It has been shown that single-walled CNT (SWCNT) interconnects can outperform $\mathrm{Cu}$ interconnects significantly, particularly for long lengths [6]-[8]. More recently, it has been further shown that multiwalled CNT (MWCNT) interconnects can also provide significant performance enhancement with respect to $\mathrm{Cu}$ interconnects [9]. As a very promising future interconnect material, besides their circuit performance in terms of delay, it is also important to investigate their high-frequency behavior.

Manuscript received February 18, 2009; revised June 18, 2009. Current version published September 23, 2009. This work was supported by the National Science Foundation under Grant CCF-0811880 and Grant CCF-0917385. The review of this paper was arranged by Editor M. Reed.

The authors are with the Department of Electrical and Computer Engineering, University of California, Santa Barbara, CA 93106 USA (e-mail: hongli@ece.ucsb.edu; kaustav@ece.ucsb.edu).

Color versions of one or more of the figures in this paper are available online at http://ieeexplore.iee.org.

Digital Object Identifier 10.1109/TED.2009.2028395

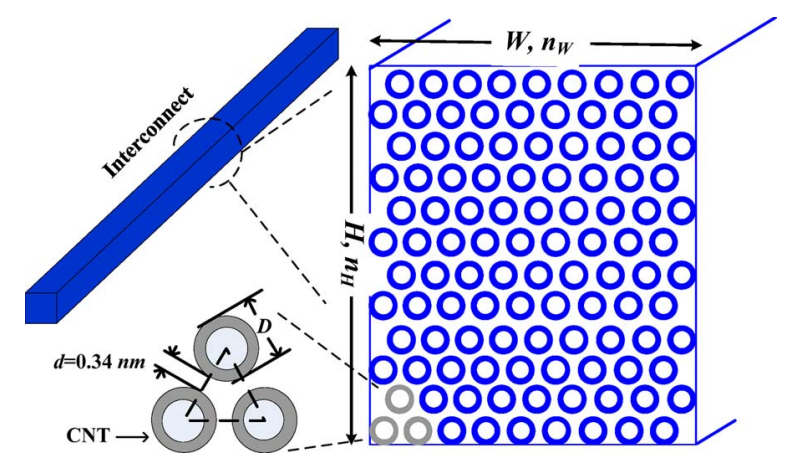

Fig. 1. Cross-sectional view of a CNT bundle with width $W$ and height $H$. The diameter of each CNT is $D$, and the interval between neighboring CNTs is $d$ (for densely packed, $d=0.34 \mathrm{~nm}$, which is the Van der Waal's gap). $n_{W}$ and $n_{H}$ are the numbers of CNTs along the width and height, respectively. For MWCNT, the diameter of the innermost shell is assumed to be $D / 2$.

This is desirable not only for future high-frequency circuit design but also due to the fact that the significant frequencies ${ }^{1}$ of digital signals in VLSI are as high as tens of gigahertz and are expected to increase further with technology scaling [10]. For traditional metallic materials, the resistance and inductance are known to be frequency dependent in the high-frequency regime due to skin and proximity effects [11], e.g., resistance increases and inductance decreases with frequency. However, the high-frequency behavior of CNT interconnects' resistance and inductance is not well understood. For CNT interconnects, a bundle structure is needed [7]-[9], as shown in Fig. 1. There are several unique properties of CNT bundle that restrict the use of conventional impedance extraction tools. First of all, a CNT bundle consists of a large number of discrete components. Second, each CNT in the bundle is a hollow cylindrical structure. Third, there is large kinetic inductance for each CNT [12], which cannot be captured by existing impedance extraction tools for conventional materials.

An existing work in the literature proposed using an "equivalent dc conductivity" to calculate the inductance of CNT bundles [13]. In this method, a realistic CNT bundle consisting of discrete conductors is replaced by a single solid conductor of identical dimension with an equivalent de conductivity.

\footnotetext{
${ }^{1}$ Significant frequency $\left(f_{s}\right)$ is the highest frequency in the frequency spectrum of a signal pulse up to which the amplitudes of the corresponding frequency components are significant. Most high-frequency digital pulses can be characterized using a significant frequency, related only to the ramp time $t_{r}\left(f_{s}=0.34 / t_{r}\right)$. Skin depth and interconnect parasitics (resistance and inductance) are also extracted at the significant frequency.
} 
Subsequently, the inductance of the CNT bundle is extracted by employing a widely used field solver, FastHenry [14], on that single "equivalent dc conductivity" solid conductor. However, such an approach has several fundamental issues that need to be justified. First, the treatment of discrete conductors as a single solid conductor is not valid for all cases. While the behavior of a very large number of discrete solid conductors may approach that of a single solid conductor, a significant difference could be induced by this treatment for small number of discrete conductors, as have been shown in [15]. Second, the realistic CNTs' hollow cylindrical structure cannot be captured by the approach in [13]. Most importantly, the "equivalent dc conductivity" method in [13] does not take into account the large kinetic inductance of CNTs when calculating the magnetic inductance. As will be shown later in this paper, kinetic inductance plays a critical role in determining the highfrequency impedance of CNTs. Without considering kinetic inductance, the impedance of CNT bundles cannot be correctly extracted in the high-frequency regime. This indicates that the inductance extraction method outlined in [13] is flawed. Since understanding the high-frequency effects in CNT interconnects is critical to further explore their applicability in high-frequency circuit design, a correct frequency-dependent impedance extraction method for CNT interconnects is highly desirable.

From an application perspective, CNT-based inductors have been proposed in several works [16], [17], although these structures cannot be easily fabricated as part of the VLSI back-end process. In [18], an SWCNT-based on-chip inductor has been analyzed based on a modified inductance extraction procedure presented in [13]. However, since the employed inductance extraction method is flawed as discussed earlier, the inductor analysis in [18] would lead to dubious quantitative results and conclusions. As has been highlighted in [15], CNT bundle interconnects have fundamentally different characteristics at high frequencies compared to conventional metals. In this paper, we not only develop a frequency-dependent impedance extraction method but also present a rigorous investigation of high-frequency effects in CNT bundle structures. Our analysis quells existing misunderstandings and provides insights that could potentially open up new vistas in RF/mixed-signal and off-chip applications for CNT interconnects.

This paper is organized as follows. Section II develops an impedance extraction method for CNT bundles, which can serve as the basis for high-frequency analysis of CNT interconnects. In Section III, high-frequency effects in CNT interconnects are discussed, where a rigorous analysis of skin effect in CNT bundle structures is performed. As an application instance, CNT-based on-chip inductors are analyzed and compared to their $\mathrm{Cu}$-based counterparts in Section IV. Finally, conclusions are drawn in Section V.

\section{IMPEDANCE ANALYSIS OF CNT INTERCONNECTS}

From the traditional analysis of 3-D metal conductors, it is well known that the resistance and inductance of interconnects are strongly dependent on the operation frequency due to skin effect and/or proximity effect [11]. Similarly, investigation of the impedance behavior of CNT interconnects at high frequen- cies is necessary to quantify their skin or proximity effect. In this section, a frequency-dependent impedance (resistance and inductance) extraction method for both SWCNT and MWCNT bundles (as shown in Fig. 1) is developed, which can serve as the basis for further high-frequency analysis of CNT interconnects.

\section{A. Impedance of a $C N T$}

The Drude model for ac electrical conductivity predicts [19]

$$
\sigma(\omega)=\frac{n e^{2} \tau}{m^{*}} \frac{1}{1+j \omega \tau}
$$

where $\tau$ is the momentum relaxation time, $n$ is the carrier density, $\omega$ is the angular frequency, and $m^{*}$ is the effective mass. For the dc case $(\omega=0)$, the conductivity reduces to $\sigma_{0}=n e^{2} \tau / m^{*}$, so that (1) can also be written as

$$
\sigma(\omega)=\sigma_{0} /(1+j \omega \tau)
$$

or in terms of resistivity

$$
\rho(\omega)=\frac{1+j \omega \tau}{\sigma_{0}}=\frac{1}{\sigma_{0}}+j \omega \frac{\tau}{\sigma_{0}}
$$

where $\sigma_{0}$ is the frequency-independent dc conductivity. For 1-D conductors, (2) reduces to conductance per unit length. If we consider the case of a 1-D conductor with only one conducting channel (two spin modes), it can be shown that (see Appendix I)

$$
\sigma_{0}^{1 D}=\frac{n_{1 D} e^{2} \tau}{m^{*}}=\frac{2 e^{2}}{h} \cdot \lambda
$$

where $n_{1 D}$ is the number of carriers per unit length and $\lambda$ is the electron mean free path. For CNTs, due to their unique conical energy dispersion near Fermi level, the velocity is constant, and the mean free path is given as

$$
\lambda=\nu_{F} \cdot \tau_{B}
$$

where $\nu_{F}$ is the Fermi velocity $\left(\sim 8 \times 10^{5} \mathrm{~m} / \mathrm{s}\right)$, and $\tau_{B}$ is the momentum backscattering time. Note that $\tau_{B}=2 \tau$ here because backscattering corresponds to momentum relaxation from $p$ to $-p$, while normal scattering corresponds to momentum relaxation from $p$ to zero [20]. Hence, (3) can be rewritten in the impedance (intrinsic) form

$$
Z_{\text {p.u.l }}=\frac{h}{2 e^{2}} \cdot \frac{1}{\lambda} \cdot(1+j \omega \tau)=\frac{h}{2 e^{2}} \cdot \frac{1}{\lambda}+j \omega \frac{h}{4 e^{2} \nu_{F}}
$$

where $h / 2 e^{2} \sim 12.9 \mathrm{k} \Omega$. The first term of (6) is the per unit length scattering resistance $\left(R_{s}\right)$

$$
R_{S}=\frac{h}{2 e^{2}} \cdot \frac{1}{\lambda}
$$

and the second term can be modeled as the inductive impedance $\left(j \omega L_{K}\right)$, where $L_{K}$ is the kinetic inductance per unit length and is equal to

$$
L_{K}=\frac{h}{4 e^{2} \nu_{F}} \sim 8 \mathrm{nH} / \mu \mathrm{m} .
$$




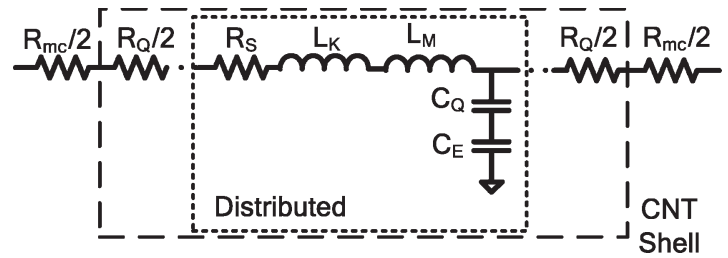

Fig. 2. Equivalent circuit model [9] of each CNT shell, where $R_{\mathrm{mc}}$ is the imperfect metal-CNT contact resistance, $R_{Q}$ is the quantum resistance, $R_{S}$ is the scattering resistance, $L_{K}$ is the kinetic inductance, $L_{M}$ is the magnetic inductance, $C_{Q}$ is the quantum capacitance, and $C_{E}$ is the electrostatic capacitance.

In reality, besides scattering resistance, there is quantum contact resistance $\left(R_{Q}\right)$ between 1-D and 3-D materials, which is also equal to $h / 2 e^{2}$ for each conducting channel. Therefore, for a CNT with $N$ conducting channels and length $L$, the total resistance and kinetic inductance are given by

$$
\begin{aligned}
& R_{\mathrm{CNT}}=\frac{1}{N}\left(R_{Q}+R_{S} \cdot L\right)=\frac{h}{2 N e^{2}}\left(1+\frac{L}{\lambda}\right) \\
& L_{K}^{\mathrm{CNT}}=\frac{L_{K}}{N} \cdot L=\frac{h}{4 N e^{2} \nu_{F}} \cdot L .
\end{aligned}
$$

It should be noted that, for conventional metals, we only consider the scattering resistance and ignore the kinetic inductance. This is due to two reasons. First of all, the momentum relaxation time for a metal is usually very small (on the order of $10^{-14} \mathrm{~s}$ ) [19], and thus, the imaginary part of (3) is negligible for frequencies less than a terahertz $(\omega \tau \ll 1)$. Second, as shown in (8), the total kinetic inductance also scales down with the number of conducting channels $N$, which usually is very large for metals, except for very small dimensions. In comparison, the momentum relaxation time for a CNT is on the order of $10^{-12}$ s or even larger $(\omega \tau \sim 1)$, and the $N$ for a CNT is small (=2 for an SWCNT). Hence, from (6) and (8b), one can observe that the kinetic inductance is significantly larger in CNTs. It is instructive to note that since the large kinetic inductance of CNTs is primarily associated with their large momentum relaxation time, the existence of such large kinetic inductance need not be restricted to CNTs in the ballistic region. The existence of large kinetic inductance in $2 \mu \mathrm{m}$ CNTs has also been experimentally verified in [21].

\section{B. Equivalent Circuit for CNT}

The aforementioned derivation of CNT impedance is consistent with many theoretical or modeling works in literature [7]-[9], [12], [22]. Based on this understanding, the equivalent circuit model of CNT has been proposed in [7]-[9] and shown in Fig. 2, where $R_{Q}, R_{S}$, and $L_{K}$ can be calculated using (8).

It has been shown that the mean free path of CNT $(\lambda)$ is proportional to the diameter [4] and can be approximated as [9]

$$
\lambda=1000 \cdot D
$$

where $D$ is the diameter of the CNT shell. Note that employing this value of $\lambda$ obtained from measurements takes into account the defects in the CNTs; the theoretical value of $\lambda$ will be much larger than that calculated using (9) [9]. The number

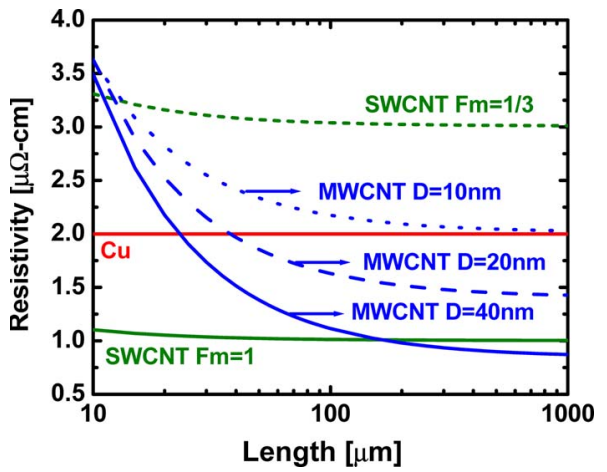

Fig. 3. Comparison of equivalent dc resistivity among SWCNT, MWCNT, and $\mathrm{Cu}$ interconnects for different diameters and lengths. $F m$ represents the fraction of metallic CNTs in the SWCNT bundle. All CNT bundles are assumed to be densely packed $(d=0.34 \mathrm{~nm})$.

$(N)$ of conducting channels equals two for metallic SWCNTs. However, for the case of large-diameter MWCNTs, $N$ of a CNT shell increases with the diameter and can be approximated by [9], [23]

$$
N_{i} \approx 6.12 \times 10^{7} \cdot D_{i}+0.425, \quad D_{i}>3 \mathrm{~nm}
$$

where $D_{i}$ is the diameter of the $i$ th shell in MWCNTs. It should be noted that the assumption in (10) is that the shells in an MWCNT have $1 / 3$ probability to be of metallic chirality and $2 / 3$ probability to be of semiconducting chirality. Since MWCNTs have many shells in parallel, the total resistance and kinetic inductance of MWCNTs can be obtained by

$$
\begin{aligned}
R_{\mathrm{MWCNT}} & =1 / \sum_{i^{\text {th }} \text { shell }} \frac{1}{R_{\mathrm{CNT}}^{i}} \\
L_{K}^{\mathrm{MWCNT}} & =\frac{L_{K}}{\sum_{i^{\text {th }} \text { shell }} N_{i}}
\end{aligned}
$$

where $R_{\mathrm{CNT}}^{i}$ is the resistance of the $i$ th shell and can be obtained from (8a), incorporating (9) and (10). The detailed model and analysis of MWCNTs can be found in [9].

As per the circuit model shown in Fig. 2, besides quantum contact resistance, there is another contact resistance $\left(R_{\mathrm{mc}}\right)$ due to the imperfect contact condition between CNTs and other materials. This resistance can range from zero to hundreds of kiloohms for different growth processes. Recently, as demonstrated in [24]-[26], the $R_{\mathrm{mc}}$ of CNTs could be very small compared to the total resistance.

In order to compare the conductance of different types of CNTs, a better way is to examine their equivalent dc resistivities based on the calculated resistances and their diameters for different types of CNT bundles, as shown in Fig. 3. It can be observed that, for the case of SWCNT bundle with metallic fraction $F m=1$ or large-diameter MWCNTs (outmost diameter $D>10 \mathrm{~nm}$ ), their dc resistivity would be lower than that of bulk $\mathrm{Cu}(\sim 2 \mu \Omega \cdot \mathrm{cm})$ for long lengths. On the other hand, for SWCNT bundles with metallic fraction $F m=1 / 3$ or MWCNTs with outmost diameter $<10 \mathrm{~nm}$, their dc resistivities are always higher than that of bulk $\mathrm{Cu}$.

In Fig. 2, it is also shown that there is magnetic inductance $\left(L_{M}\right)$ associated with CNTs, which is the traditional inductive 


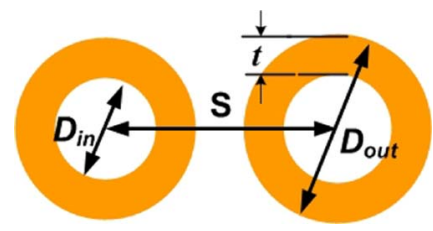

Fig. 4. Geometry of two adjacent CNTs with outer diameter $D_{\text {out }}$, inner diameter $D_{\text {in }}$, and center-to-center distance (or pitch) $S$. The thickness of the hollow cylinder $t=\left(D_{\text {out }}-D_{\text {in }}\right) / 2$, which is assumed to be the diameter of carbon atom $(0.142 \mathrm{~nm})$ for SWCNTs and $D_{\text {out }} / 4$ for MWCNTs.

component due to the electromagnetic (EM) field. The magnetic inductance consists of self-inductance $\left(L_{\text {self }}\right)$ and mutual inductance $(M)$. Since CNT is a cylindrical structure, its selfinductance and mutual inductance can be obtained from [27] by employing the concepts of geometric mean distance (GMD) and arithmetic mean distance (AMD) as follows (see Appendix II for details):

$$
\begin{aligned}
L_{\text {self }} & =\frac{\mu_{0}}{2 \pi} L \cdot\left[\ln \frac{2 L}{G M D}-1+\frac{A M D}{L}\right] \\
M & =\frac{\mu_{0}}{2 \pi} \cdot L \cdot\left[\ln \left(\frac{L}{S}+\sqrt{1+\frac{L^{2}}{S^{2}}}\right)-\sqrt{1+\frac{S^{2}}{L^{2}}}+\frac{S}{L}\right]
\end{aligned}
$$

where $L$ is the length of CNT, and $S$ is the GMD between two CNTs (equals the center-to-center distance between two CNTs due to their cylindrical structure), as shown in Fig. 4. For hollow concentric cylindrical structures with innermost and outmost diameters of $D_{\text {in }}$ and $D_{\text {out }}$, respectively, their $G M D$ can be extrapolated from [28] for an arbitrary ratio of $D_{\text {in }} / D_{\text {out }}$ as follows:

$$
\begin{aligned}
\ln G M D & =\ln D_{\text {out }}-\ln \chi \\
\ln \chi & =0.1\left(a-b \gamma-c \gamma^{2}+d \gamma^{3}\right) \\
\gamma & =D_{\text {in }} / D_{\text {out }} \quad a=2.51 \\
b & =0.31 \quad c=3.81 \quad d=1.61 .
\end{aligned}
$$

The third term (12) is usually negligible for long lengths, where $A M D$ can be approximated as

$$
A M D \approx \frac{2 D_{\text {out }}}{\pi} .
$$

For typical geometries, the values of (12) and (13) are on the order of $\mathrm{pH} / \mu \mathrm{m}$, which is much smaller than the kinetic inductance counterpart.

\section{Impedance Extraction of CNT Bundle}

The self-impedance and mutual impedance of each CNT in Fig. 4 can be expressed as

$$
\begin{aligned}
Z_{\text {self }} & =R_{\mathrm{CNT}}+j \omega\left(L_{K}^{\mathrm{CNT}}+L_{\text {self }}\right) \\
Z_{\text {mutual }} & =j \omega M
\end{aligned}
$$

where $R_{\mathrm{CNT}}, L_{K}^{\mathrm{CNT}}, L_{\mathrm{self}}$, and $M$ can be obtained from the previous section, depending on the type of CNT. Note that the self-impedance of each CNT consists of resistive and inductive (both kinetic and magnetic) impedances. On the other hand,

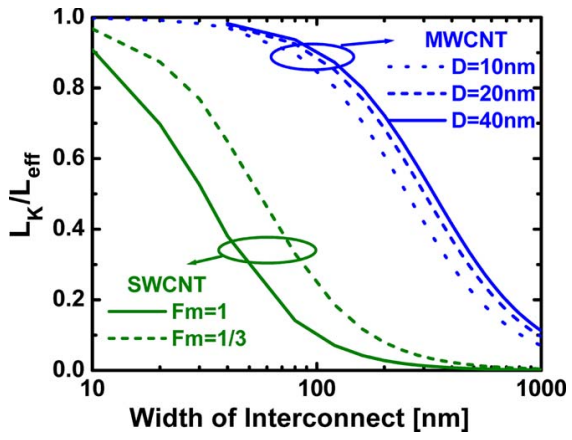

Fig. 5. Ratio of kinetic inductance to total effective inductance of SWCNT (with different metallic fractions $F m$ ) and MWCNT (with different diameters) bundles as a function of interconnect width. The interconnect height is set equal to the width; the interconnect length is $500 \mu \mathrm{m}$.

mutual impedance only consists of magnetic component since there is no mutual kinetic inductance. The intertube resistance among CNTs in a bundle has been shown to be on the order of megaohms [29] and their effect can be ignored. Employing (16) and (17) into a CNT bundle structure, we can derive the impedance matrix of the CNT bundle as

$$
\left[Z_{\text {matrix }}\right]=\left[\begin{array}{cccc}
Z_{\text {self }}^{1} & j \omega M^{21} & \cdots & j \omega M^{n 1} \\
j \omega M^{21} & Z_{\text {self }}^{2} & \cdots & j \omega M^{n 2} \\
\vdots & \vdots & \ddots & \vdots \\
j \omega M^{n 1} & j \omega M^{n 2} & \cdots & Z_{\text {self }}^{n}
\end{array}\right]
$$

where $Z_{\text {self }}^{i}$ is the self-impedance of the $i$ th nanotube, and $M^{i j}$ is the mutual inductance between the $i$ th and $j$ th nanotubes in the bundle. Note that, by employing (12) and (13), the impedance matrix (18) has not only considered the realistic hollow cylindrical structure but also taken into account the discreteness of the CNTs in a bundle. Having this impedance matrix, the total effective impedance of the bundle can be obtained for a given frequency $\omega$ by solving the matrix equation $[V]=[Z][I]$, where the current matrix $[I]$ can be extracted for a given voltage $V_{0}$ across the bundle. Note that, for very large $[Z]$ matrices, one can employ judicious partitioning techniques (considering current distributions in the bundle) for calculating $[I]$. The effective total impedance can be obtained by

$$
Z_{\text {eff }}=V_{0} / \operatorname{sum}[I]
$$

where $\operatorname{sum}[I]$ is the sum of the currents in each CNTs in the bundle for voltage $V_{0}$. The total effective resistance and inductance can be obtained as

$$
\begin{aligned}
& R_{\text {eff }}=\operatorname{real}\left(Z_{\text {eff }}\right) \\
& L_{\text {eff }}=\operatorname{imag}\left(Z_{\text {eff }}\right) / \omega .
\end{aligned}
$$

Since the impact of kinetic inductance has raised some concerns in terms of its significance [6], [21], its fraction in the total effective inductance of CNT interconnects for different cross sections has been analyzed at low frequency (where inductance will not change with frequency) using our method and shown in Fig. 5. As can be observed, kinetic inductance forms a significant fraction of total inductance only when the width of SWCNT interconnects is smaller than $100 \mathrm{~nm}$. However, it is 

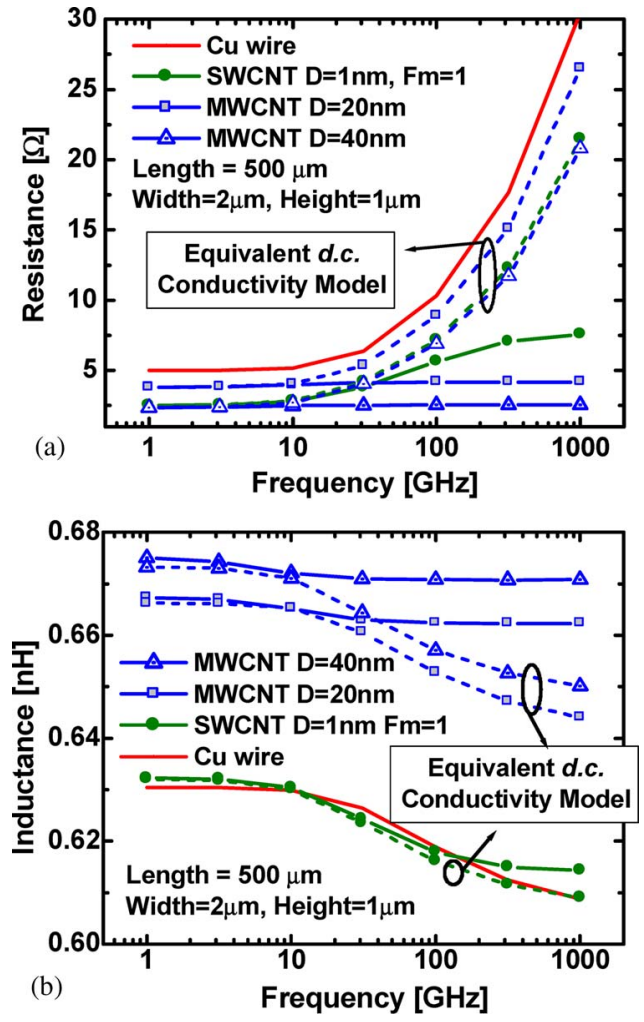

Fig. 6. Total effective (a) resistance and (b) inductance of SWCNT and MWCNT bundles with different diameters as a function of frequency, compared with identical cross-sectional $\mathrm{Cu}$ wire. For the $\mathrm{Cu}$ wire, it is simulated using the field solver FastHenry [14]. Fm indicates the fraction of metallic SWCNTs in the SWCNT bundle. The broken lines are the results obtain for the CNTs from the "equivalent dc conductivity" method in [13].

much more significant in the case of MWCNT bundles. This is because the number of conducting channels $(N)$ of MWCNT bundles is small compared to that of SWCNT bundles for a given cross section [9], and the total kinetic inductance scales down linearly with $N$, as shown in (8b) and (11b).

\section{High-FREQUENCY ANALYSIS OF CNT INTERCONNECTS}

\section{A. High-Frequency Impedance of CNT Interconnects}

Based on the impedance extraction procedure outlined earlier, the frequency-dependent effective resistance and inductance of CNT bundles can be obtained. Fig. 6 shows the resistance and inductance of CNT interconnect, as a function of frequency, as compared with those of a $\mathrm{Cu}$ wire. The interconnect width and height are assumed to be $2 \mu \mathrm{m}$ and $1 \mu \mathrm{m}$, respectively. It can be observed that the resistance of $\mathrm{Cu}$ wire increases dramatically in the high-frequency region due to significant skin effect. However, it is interesting to observe that the resistance/inductance of CNT bundles increase/decrease by a small amount and saturate at high frequencies, implying reduced skin effect. Particularly for MWCNTs, their resistance almost remains unchanged (increase $<8 \%$ ), implying negligible skin effect.

For 500- $\mu \mathrm{m}$ length, all the CNT cases shown in Fig. 6 (i.e., SWCNT with $D=1 \mathrm{~nm}$ and $F m=1$, and MWCNT with $D=20 \mathrm{~nm}$ or $40 \mathrm{~nm}$ ) exhibit smaller resistivity than that of

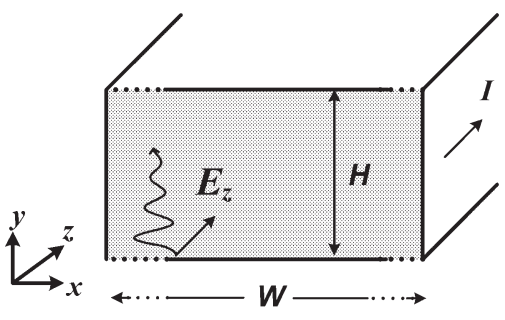

(a)

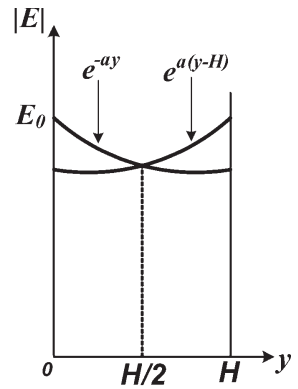

(b)
Fig. 7. (a) Conductor with height $H$ and semi-infinite width $(W \gg H)$ and length. The EM wave propagates in the $y$-direction (with the current and $E$-field in the $z$ direction). (b) Schematic illustration of the decay of electric field inside the conductor along the height ( $y$ direction).

$\mathrm{Cu}$ (as shown in Fig. 3), which implies that, if they followed the traditional physics of skin effect, their skin depths would be smaller than that of $\mathrm{Cu}$, and therefore, the skin effect would have been more significant. However, the resistance and inductance curves in Fig. 6 imply that, contrary to traditional wisdom, the skin effect in a CNT bundle is much less significant. The reason for this unusual result can be attributed to the presence of large kinetic inductance in each CNT, which could be more than two orders larger than the magnetic component or, in other words, as discussed in Section II-A, due to large momentum relaxation time in CNTs.

\section{B. Skin Effect in CNT Bundle Structures}

In order to explain the aforementioned reduced skin effect in CNT bundles, a rigorous investigation using Maxwell's EM theory is preferred. The full-wave analytical modeling of EM wave transmission for a single CNT in antenna application has been shown in [22]. However, a fully analytical treatment of EM wave ( $E$-field) attenuation inside a CNT bundle, directly from the Maxwell's equations, is nontrivial since it consists of a large number of discrete conductors. In order to make it feasible, we treat the CNT bundle as a homogenous material with longitudinal complex (ac) conductivity, as shown in (2), and investigate the skin effect and high-frequency properties for this CNT material.

For a good conductor, where displacement current is negligible compared to the conduction current (e.g., $\omega \varepsilon \ll \sigma$ ), one can derive (see Appendix III) the following relation from the Maxwell's equations:

$$
\nabla^{2} \vec{E}=j \omega \mu \sigma \vec{E}
$$

where $\mu$ and $\sigma$ are the permeability and conductivity of the material, respectively. Considering a semi-infinite material [as shown in Fig. 7(a)] with height $H$, width $W(W>>H)$, and with current (or $E$-field) in the $z$ direction, the electrical field propagation in the direction that is perpendicular to the $x-z$ surface ( $y$ direction) will follow (22), whose 1-D form is

$$
\frac{d^{2} E_{z}}{d y^{2}}=j \omega \mu \sigma E_{z}=\Gamma^{2} E_{z}
$$


where

$$
\Gamma=\sqrt{j \omega \mu \sigma}=\alpha+j \beta
$$

and $E_{z}$ can be solved as

$$
E_{z}=E_{0} e^{-\Gamma y}=E_{0} e^{-\alpha y-j \beta y} .
$$

The first exponential component $e^{-\alpha y}$ represents the exponential decay of electrical field in $y$ direction inside the conductor, as shown in Fig. 7(b). The second exponential component $e^{-j \beta y}$ represents the E-field propagation along $y$ direction. Substituting (2) into (24), $\alpha$ and $\beta$ are obtained as

$$
\begin{aligned}
& \alpha=\sqrt{\frac{\omega \mu \sigma_{0}}{2}} \cdot \sqrt{\frac{1}{(\omega \tau)^{2}+1}\left[\sqrt{(\omega \tau)^{2}+1}+\omega \tau\right]} \\
& \beta=\sqrt{\frac{\omega \mu \sigma_{0}}{2}} \cdot \sqrt{\frac{1}{(\omega \tau)^{2}+1}\left[\sqrt{(\omega \tau)^{2}+1}-\omega \tau\right]} .
\end{aligned}
$$

The skin depth $\delta$ is defined as $1 / \alpha$, which is

$$
\delta=\sqrt{\frac{2}{\omega \mu \sigma_{0}}} \cdot \sqrt{\left[(\omega \tau)^{2}+1\right] \cdot\left[\sqrt{(\omega \tau)^{2}+1}-\omega \tau\right]} .
$$

For conventional metals ( $\tau$ is very small or $\omega \tau \ll 1)$, (28) reduces to the classical skin depth

$$
\delta_{c}=\sqrt{2 / \omega \mu \sigma_{0}} .
$$

For CNTs, the momentum relaxation time can be obtained by

$$
\tau=\frac{\tau_{B}}{2}=\frac{\lambda}{2 \nu_{F}} \approx \frac{500 D}{\nu_{F}}
$$

Hence, the equivalent skin depths of different types of CNT bundles can be calculated using (28) and (30) and are shown in Fig. 8(a).

From Fig. 8(a), it can be observed that the skin depth of $\mathrm{Cu}$ wire continues to reduce with frequency, whereas the skin depths of CNTs saturate after certain frequencies due to the existence of large kinetic inductance or large momentum relaxation time $\tau$. The value of this saturation frequency depends on the momentum relaxation time $\tau$. Larger diameter CNTs have larger $\tau$ and will saturate earlier. It is interesting to find that MWCNTs will not only start saturating at a relatively lower frequency but will also have relatively larger saturated skin depths. MWCNTs with diameters of $20 \mathrm{~nm}$ and $40 \mathrm{~nm}$ have similar saturated skin depths. Considering the fact that MWCNTs could have comparable circuit performance to that of SWCNTs [9], it can be expected that MWCNTs would have significant advantages in high-frequency applications.

We further evaluate the high-frequency resistance of CNT materials, considering the structure shown in Fig. 7(a). Since $W \gg H$, the skin effect is determined by the height $H$. The
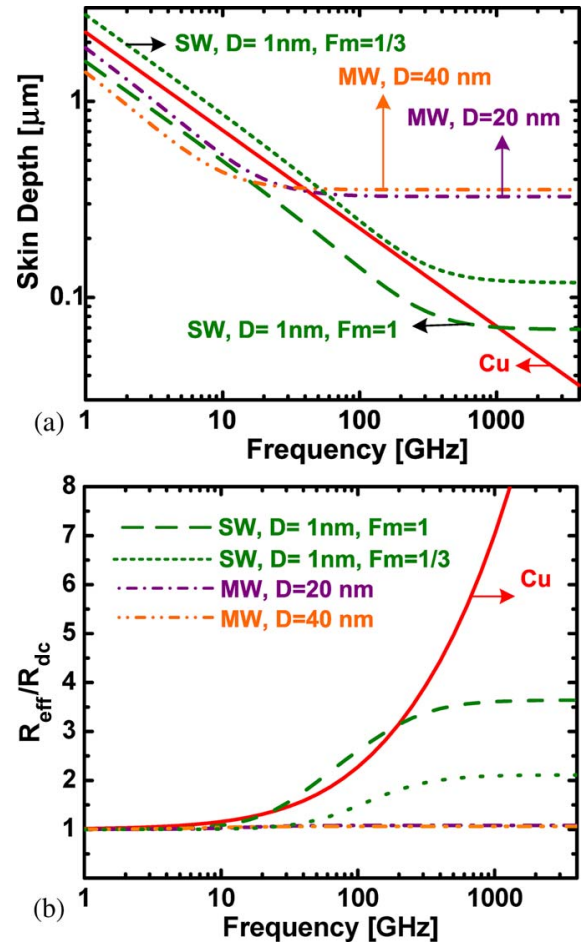

Fig. 8. (a) Skin depth of different types of CNT material, as well as $\mathrm{Cu}$, as a function of frequency. (b) Ratio of high-frequency effective resistance with respect to dc resistance for different types of CNT bundles and $\mathrm{Cu}$.

total current of the conductor shown in Fig. 7(a) can be calculated as

$$
\begin{aligned}
I & =\int J \cdot d A=\int \sigma(\omega) \cdot E \cdot d A \\
& =2 W \cdot \int_{0}^{H / 2} \sigma(\omega) \cdot E_{0} \cdot\left[e^{-\Gamma y}+e^{\Gamma(y-H)}\right] d y \\
& =W \cdot \sigma(\omega) \cdot E_{0} \cdot \frac{2}{\Gamma}\left(1-e^{-\Gamma H}\right)
\end{aligned}
$$

where $E_{0}$ is the incident electric field, and $\Gamma$ is given by (24). From Fig. 7(b), one can derive the E-field on the $x-z$ surface of conductor, $E_{\text {surf }}$, as

$$
E_{\text {surf }}=E_{0}\left(1+e^{-\Gamma H}\right)
$$

Therefore, the effective resistance per unit length can be calculated as:

$$
R_{\mathrm{eff}}=\operatorname{Re}\left(\frac{E_{\text {surf }}}{I}\right)=\operatorname{Re}\left(\frac{\Gamma \cdot\left(1+e^{-\Gamma H}\right)}{2 \cdot W \cdot \sigma(\omega) \cdot\left(1-e^{-\Gamma H}\right)}\right) .
$$

Fig. 8(b) shows the ratio of $R_{\text {eff }}$ with respect to dc resistance as a function of frequency, for $H=1 \mu \mathrm{m}$. It can be observed that the resistance of $\mathrm{Cu}$ increases dramatically at high frequencies, whereas the resistance of CNTs saturates at high frequencies. Particularly, MWCNTs exhibit only a small increase $(<9 \%)$. It should be noted that this slight increment in the resistance of MWCNTs is for the case with conductor 


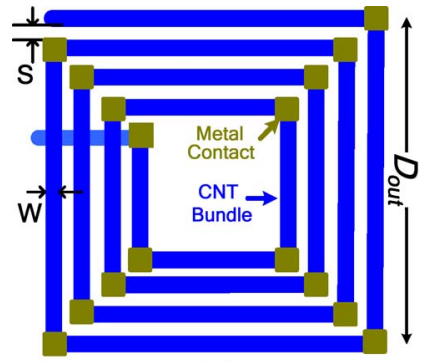

(a)

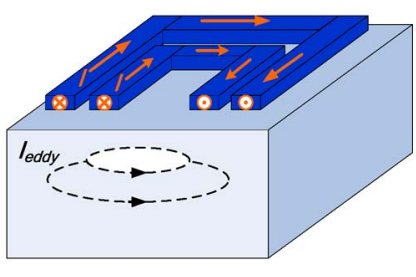

(b)

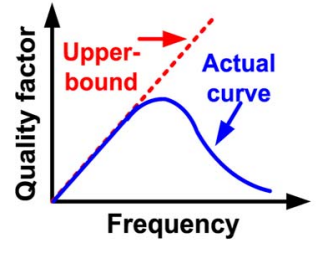

(a)

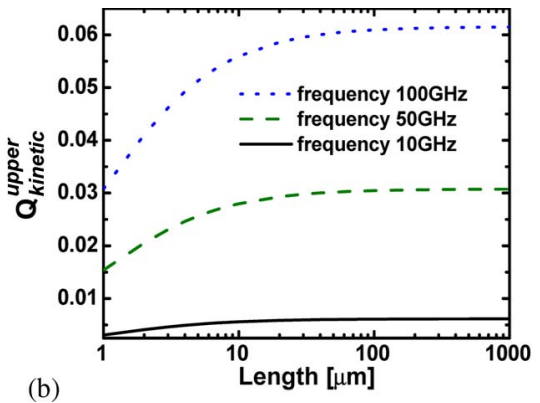

(b)

Fig. 9. (a) Schematic view of a four-turn planar spiral CNT inductor. At each corner, there is a metal contact to connect CNT bundles. (b) Schematic view of substrate eddy currents (in the opposite direction of inductor currents).

height $H=1 \mu \mathrm{m}$; if the height is larger, the resistance increase in MWCNTs will be greater than this amount.

The aforementioned rigorous skin effect analysis of CNT material is in good agreement with our impedance extraction results shown in Fig. 6. For comparison, Fig. 6 also shows the results obtained from a previous CNT impedance extraction "equivalent dc conductivity" method [13] using FastHenry [14]. At low frequencies, the results using the method in [13] are in agreement with those obtained using the methodology presented in this paper. However, as can be observed, because the kinetic inductance is not included in that "equivalent dc conductivity" method, the high-frequency properties of CNT bundles cannot be correctly captured by that method.

From the aforementioned analysis, it can be concluded that the large kinetic inductance in CNTs has two effects. The first one is that it increases the overall inductance value. However, this increment becomes small (particularly for SWCNTs) for large bundle size due to the fact that the total kinetic inductance scales down with the number of channels or number of tubes. This can be observed from Fig. 5. The second effect is more subtle and only becomes significant in high-frequency region, which leads to the reduced skin effect in CNT bundles, as has been discussed in this section.

\section{CNT-BASED ON-CHIP INDUCTOR}

The high-frequency effect in CNT bundles as discussed in the previous section is very exciting for high-frequency applications. For high-frequency circuit design, one of the important concerns is that the conductor loss increases dramatically due to increasing resistance at high frequencies (due to skin and proximity effects). Since the resistance of CNT interconnects increases by a smaller amount or remains almost unchanged at high frequencies, the high-frequency performance of the circuit can be enhanced significantly by employing CNT interconnects. In this section, on-chip inductor is analyzed as an instance to demonstrate the promising high-frequency performance of CNT interconnects.

One of the proposed structures for CNT-based spiral inductor is shown in Fig. 9(a), where a metal block at each corner is employed as contact for CNT bundles along perpendicular directions. The four-turn inductor shown in Fig. 9(a) is for lowfrequency applications $(<10 \mathrm{GHz})$. For high-frequency applications, inductors with fewer turns are preferred (a 0.75-turn

Fig. 10. (a) Schematic view of $Q$ factor curve of a spiral inductor. The upper bound of $Q$ factor is $\omega L_{S} / R_{S}$, as shown by the broken line. Due to the presence of parasitic capacitance, conductor and substrate loss, the actual $Q$ factor curve drops down at high frequencies. (b) Upper-bound of $Q$ factor introduced by kinetic inductance of CNTs as a function of frequency and length.

inductor will be analyzed later). A similar structure, in which CNT bundle is grown along perpendicular directions from a common block, has been fabricated in [30], which indicates that the structure shown in Fig. 9(a) can be possibly fabricated in the near future. It should be noted that, although there are still a lot of challenges remaining in the CNT interconnect fabrication and integration area, the CNT interconnect fabrication technology is progressing rapidly. For example, the growth temperature of CNT via has been recently lowered to $365^{\circ} \mathrm{C}$, and with this low temperature growth, the integration of CNT via structure in ultralow $k$ dielectric $(k=2.6)$ with good reliability performance has also been demonstrated [31]. All of these progresses indicate the technological feasibility of CNT interconnects.

For inductor design, quality factor $(Q)$ is a very important performance metric. It is defined as

$$
Q=2 \pi \frac{E_{\text {stored } / \text { cycle }}}{E_{\text {lost } / \text { cycle }}}=\omega \frac{W_{\text {mag }}-W_{\text {elect }}}{P_{\text {diss }}}
$$

where $E_{\text {stored/cycle }}$ is the energy stored in the system per cycle, while $E_{\text {lost } / \text { cycle }}$ is the energy lost per cycle. $P_{\text {diss }}$ represents the average power dissipated by the system, and $W_{\text {mag }}$ and $W_{\text {elect }}$ represent the averages of the stored magnetic and electric energies, respectively. The lower the loss of a device, the higher the $Q$ factor. From an inductor perspective, $Q$ factor can also be regarded as a measure of the ratio of the desired quantity, related to the inductive reactance $(=\omega L)$, to the undesired quantity (resistance). High- $Q$-factor inductors are more desirable since higher $Q$ factor indicates not only lower loss but also higher frequency stability, as well as lower phase noise, which is particularly important for oscillators [32]. For an ideal inductor with only series inductance $L_{S}$ and resistance $R_{S}$ but no capacitance (i.e., $W_{\text {elect }}=0$ ), its $Q$ factor is given by $\omega L_{S} / R_{S}$, i.e., linear with frequency. In reality, the $Q$ factor of an inductor will not be linear with frequency. The typical $Q$ factor versus frequency curve of an inductor is shown in Fig. 10(a), where the ideal $Q$ factor $\left(\omega L_{S} / R_{S}\right)$ serves as an upper bound of the $Q$ factor. At low frequencies, $Q$ usually follows the trend of $\omega L_{S} / R_{S}$. As frequency increases, capacitive and magnetic coupling with substrate become more and more significant, which increases energy dissipation. In addition, the skin/proximity effects of the conductor also increase the 


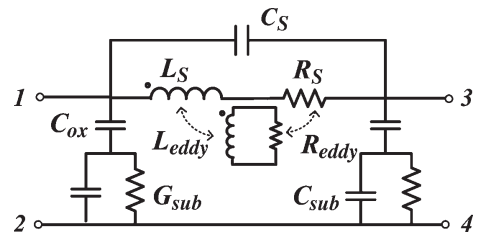

Fig. 11. Equivalent 11-element $\pi$ model for on-chip spiral inductors. $L_{S}$ and $R_{S}$ are the frequency-dependent series inductance and resistance, respectively. For CNT, $L_{S}$ and $R_{S}$ are calculated using the method outlined in Section II, which includes $L_{K}$ and $R_{Q}$, respectively. $L_{e d d y}$ and $R_{e d d y}$ capture the eddy current effects in the substrate. $C_{S}, C_{o x}$, and $C_{s u b}$ represent the inter-turn conductor capacitance, oxide capacitance and substrate capacitance, respectively.

resistive loss in the inductor at high frequencies. As a result, $Q$ factor starts to fall off at high frequencies, as shown schematically in Fig. 10(a).

In this paper, the $Q$ factor is calculated using a traditional method

$$
Q=-\frac{\operatorname{imag}\left(Y_{11}\right)}{\operatorname{real}\left(Y_{11}\right)}
$$

where $Y_{11}$ is the input admittance of the inductor network (across ports 1 and 2 with ports 3 and 4 shorted, in Fig. 11).

\section{A. Contribution of Kinetic Inductance to $Q$ Factor}

Since CNTs have very large kinetic inductance, one natural question that arises is as follows: Will this large inductance increase the $Q$ factor of CNT-based inductors accordingly? For CNT-based inductors, the $Q$ factor can be considered to be consisting of two parts: 1) a magnetic inductance component $\left(Q_{\text {magnetic }}\right)$, which is the same as in a conventional inductor and 2) a kinetic inductance component $\left(Q_{\text {kinetic }}\right)$, which is relevant for mesoscopic (low-dimensional) materials, such as CNTs. As shown in Fig. 10(a), if only the upper bound $\left(Q_{\text {total }}^{\text {upper }}\right)$ of $Q$ factor is considered, the kinetic inductance component of the $Q$ factor can be obtained by

$$
\begin{aligned}
Q_{\mathrm{total}}^{\text {upper }} & =\omega \frac{L_{\text {total }}}{R}=\omega \frac{\left(L_{\text {magnetic }}+L_{\text {kinetic }}\right)}{R} \\
& =Q_{\text {magnetic }}^{\text {upper }}+Q_{\text {kinetic }}^{\text {upper }} \\
Q_{\text {kinetic }}^{\text {upper }} & =\frac{\omega L_{K}^{\text {bundle }}}{R_{\text {bundle }}}=\frac{\omega L_{K} \cdot L / N_{\mathrm{ch}}}{R_{Q} \cdot(1+L / \lambda) / N_{\mathrm{ch}}} \\
& =\frac{\omega L_{K}}{R_{Q}(1 / L+1 / \lambda)}
\end{aligned}
$$

where the superscript "upper" denotes the upper bound value of $Q, L$ is the length, $\lambda$ is the mean free path of CNT, $N_{\mathrm{ch}}$ is the total number of conducting channels of the bundle, and $L_{K}$ is given by (7b). For the typical value of $\lambda \sim 1 \mu \mathrm{m}$, Fig. 10(b) shows the $Q_{\text {kinetic }}^{\text {upper }}$ as a function of frequency and length. It is observed that, although the kinetic inductance of each CNT has large value, $Q_{\text {kinetic }}$ is almost negligible $(<0.065$ at $100 \mathrm{GHz}$ ). Therefore, in order to achieve high- $Q$ inductors (preferably $>10$ in realistic applications), the magnetic-fieldinduced inductance, being the main contributor, should be maximized. Since the magnetic inductance is dependent on the geometry and area, it can be expected that CNT-based inductors would require similar area as conventional metal inductors.

\section{B. CNT-Based Inductor Evaluation}

In order to model on-chip inductors, the widely used $\pi$ model [33] is modified to include the effect of substrate (by adding $L_{e d d y}$ and $R_{e d d y}$ components), as shown in Fig. 11. $L_{S}$ and $R_{S}$ are the series frequency-dependent inductance and resistance, respectively, of CNT interconnects without considering the substrate effect. For CNT-based inductors, $L_{S}$ and $R_{S}$ can be obtained by employing the impedance extraction procedure discussed in Section II, while for Cu-based inductors, they can be extracted using FastHenry [14]. At high frequencies, the eddy currents induced in the substrate will significantly decrease the performance of the inductor. These eddy currents are in an opposite direction with respect to the current of the inductor, as schematically shown in Fig. 9(b). They will not only reduce the effective magnetic energy stored in the system but also increase energy dissipation due to substrate loss. In order to capture the effect of the substrate, the complex image theory [34] is employed. Using the complex image theory, the substrate effect can be simplified as conductors with their images placed at a distance of $h_{\text {eff }}$ (complex number), without the presence of the substrate. The distance between conductor and its image $h_{\text {eff }}$ can be calculated by

$$
h_{\mathrm{eff}}=h_{\mathrm{ox}}+(1-j) \frac{\delta_{\mathrm{sub}}}{2} \tanh \left[\frac{(1+j) h_{\mathrm{si}}}{\delta_{\mathrm{sub}}}\right]
$$

where $h_{\mathrm{ox}}$ is the oxide thickness, $h_{\mathrm{si}}$ is the substrate thickness, and $\delta_{\mathrm{sub}}$ is the skin depth of substrate $\left(\delta_{\mathrm{sub}}=\sqrt{2 \rho_{\mathrm{sub}} / \mu \omega}\right)$. Aside from resistance and inductance, there are several capacitances in the on-chip inductor model, as shown in Fig. 11, such as inter-turn (including turn to underpass) capacitance $\left(C_{S}\right)$, capacitance between inductor-conductors and substrate (oxide capacitance $\left.C_{\mathrm{ox}}\right)$, and substrate capacitance $\left(C_{\text {sub }}\right)$. All these capacitances can be calculated using the distributed capacitance model [35].

Based on the model in Fig. 11, the performance of CNTbased inductors can be analyzed and compared to that of $\mathrm{Cu}$ inductors. Here, the inductor considered is a four-turn planar spiral inductor, which has outermost diameter $D_{\text {out }}=200 \mu \mathrm{m}$, conductor width $W=8 \mu \mathrm{m}$, conductor thickness $H=2 \mu \mathrm{m}$, conductor spacing $S=1 \mu \mathrm{m}$, and oxide and substrate thicknesses of $10 \mu \mathrm{m}$ and $300 \mu \mathrm{m}$, respectively. The $Q$ factor analysis results are shown in Fig. 12 for different types of CNTs and substrate resistivities $\left(\rho_{\text {sub }}=10 \Omega \cdot \mathrm{cm}\right.$ and $\rho_{\text {sub }}=$ $0.01 \Omega \cdot \mathrm{cm}$ ). It can be observed that, for low-loss (highresistivity; $10 \Omega \cdot \mathrm{cm}$ ) substrate [see Fig. 12(a)], the maximum $Q$ factor of CNT-based inductor can be $\sim 140 \%$ (2.4 X) higher than that of $\mathrm{Cu}$ inductor (for densely packed MWCNTs with $D=40 \mathrm{~nm}$ ). This significant enhancement in $Q$ factor arises not only because of the lower dc resistance of CNTs but also because of the reduced skin effect in CNT interconnects. This is clear from examining the cases of MWCNTs with $D=10 \mathrm{~nm}$ or SWCNT with metallic fraction $F m=1 / 3$. Both of these cases with CNTs have larger resistivity than $\mathrm{Cu}$, as shown in Fig. 3. However, inductors based on these materials have better or comparable $Q$ factors to that of $\mathrm{Cu}$-based inductor. This indicates that reduced skin effect in CNT bundles has significant positive impact on the performance of inductors. For 

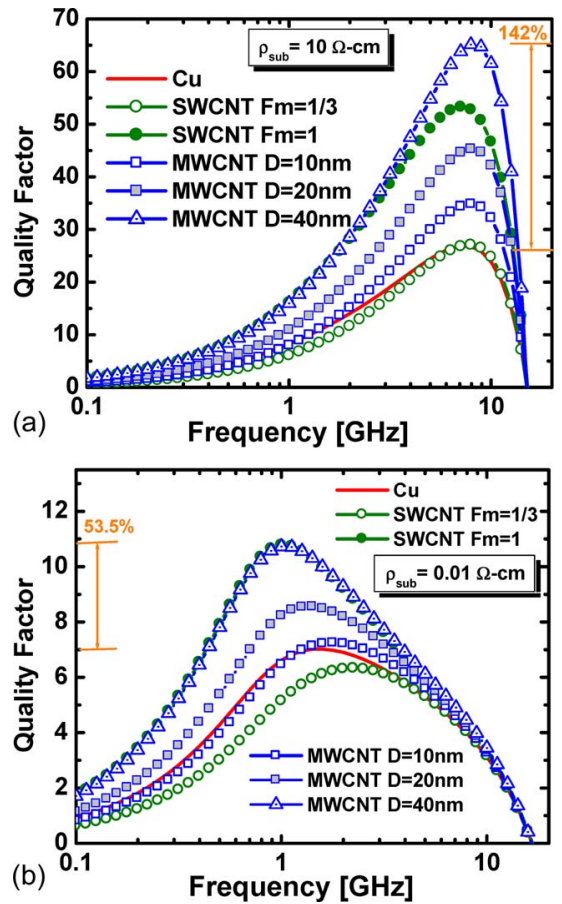

Fig. 12. Quality factor of four-turn inductors based on $\mathrm{Cu}$, SWNCT, and MWCNT interconnects as a function of frequency, for (a) low-loss substrate $\rho=10 \Omega \cdot \mathrm{cm}$ and (b) high-loss substrate $\rho=0.01 \Omega \cdot \mathrm{cm}$. All inductors have the outmost diameter of $200 \mu \mathrm{m}$, four turns, wire width $W=8 \mu \mathrm{m}$, wire thickness $H=2 \mu \mathrm{m}$, and wire spacing $S=1 \mu \mathrm{m}$. Underlying oxide and substrate thicknesses are assumed to be $10 \mu \mathrm{m}$ and $300 \mu \mathrm{m}$, respectively. $\mathrm{SiO}_{2}$ is assumed for all insulators.

high-loss (low-resistivity) substrate [see Fig. 12(b)], the impact of substrate eddy currents becomes significant, and the benefit of CNT's high-frequency properties gets eclipsed by large substrate eddy current loss, which reduces the maximum $Q$ factor, as well as the corresponding frequency from $\sim 10$ to $1 \mathrm{GHz}$ (so that the advantage of reduced skin effect in CNT interconnects is less significant). Nevertheless, CNT-based inductors can still offer better $Q$ factors [maximum enhancement is $53 \%(1.5 \mathrm{X})$, as shown in Fig. 12(b)] than that of $\mathrm{Cu}$ except for the case of SWCNT with $F m=1 / 3$.

\section{Ultrahigh Frequency Applications}

The previous inductor example is for frequencies in the range of $1-10 \mathrm{GHz}$ applications. Recently, ultrahigh frequency (tens of gigahertz) RF circuits have attracted lots of interests, such as for 24- [36] or $60-\mathrm{GHz}$ [37] applications. In this ultrahigh frequency region, the advantage of CNT's reduced skin effect is expected to be more prominent.

The inductors for these ultrahigh frequency RF circuits are usually designed to have relatively small number of turns and, hence, small area, since the inductance required is small to support such high-frequency operation. Fig. 13(a) shows one possible inductor design that has only $3 / 4$ turn, an outer diameter of $100 \mu \mathrm{m}$, and conductor width and thickness of $5 \mu \mathrm{m}$ and $1 \mu \mathrm{m}$, respectively. Fig. 13(b) and (c) shows the $Q$ factor of such inductors at several tens of gigahertz application for different types of CNT bundles and Cu wire. It can be observed that, for low-loss substrate [Fig. 13(b)], the maximum $Q$ factor of inductor can be increased by as much as $230 \%$ (3.3 X) by

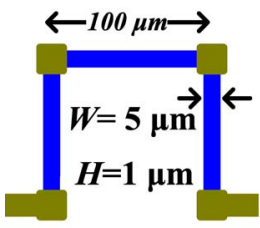

(a)
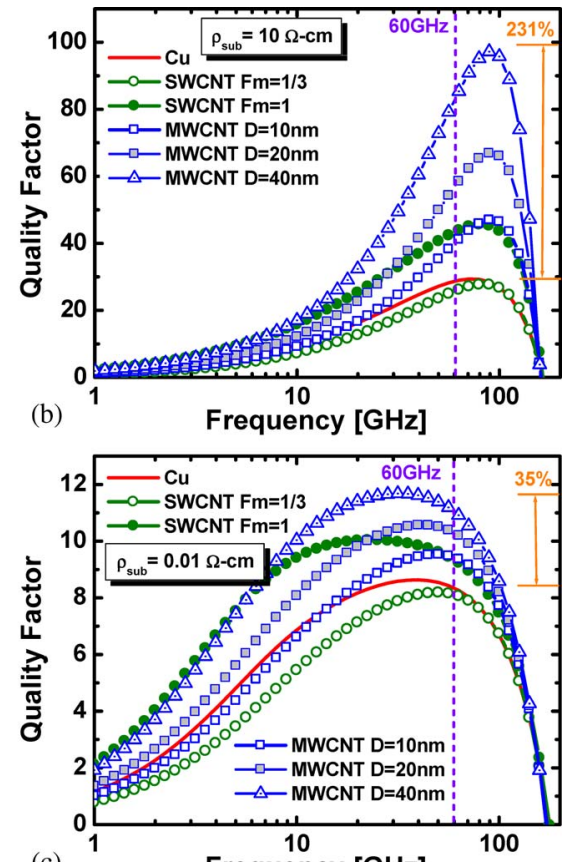

(c)

Frequency [GHz]
Fig. 13. (a) Structure of ultrahigh frequency inductor, which is 0.75-turn and has an outer diameter of $100 \mu \mathrm{m}, 5-\mu \mathrm{m}$ width, and 1- $\mu \mathrm{m}$ thickness. Quality factor of inductors based on $\mathrm{Cu}$, SWNCT, and MWCNT interconnects as a function of frequency for (b) low-loss $(\rho=10 \Omega \cdot \mathrm{cm})$ and (c) high-loss $(\rho=0.01 \Omega \cdot \mathrm{cm})$ substrate. Underlying $\mathrm{SiO}_{2}$ and substrate thicknesses are assumed to be $10 \mu \mathrm{m}$ and $300 \mu \mathrm{m}$, respectively.

going from $\mathrm{Cu}$ to CNTs. The higher $Q$ factor enhancement in this case, compared to the one in Fig. 12, indeed implies that CNT interconnects have more significant advantage at higher frequencies. For low-loss substrate, the SWCNTs will be better than $\mathrm{Cu}$ if $\mathrm{Fm}>1 / 3$, and all MWCNTs outperform $\mathrm{Cu}$. In fact, even the worst case MWCNT considered here (with a diameter of $10 \mathrm{~nm}$ ) can have comparable performance to that of the best case SWCNT $(F m=1)$. This again highlights the advantages of employing MWCNTs in high-frequency applications. For high-loss substrate [Fig. 13(c)], CNT would also have better performance than $\mathrm{Cu}$ except for the case of SWCNT with $F m=1 / 3$. The maximum $Q$ factor enhancement in high-loss substrate is $35 \%(1.35 \mathrm{X})$. For the sake of better understanding, the series resistance and inductance $\left(R_{S}\right.$ and $\left.L_{S}\right)$ of this 0.75 -turn inductor are further shown in Fig. 14, from which the reduced skin effect in CNT (particularly in MWCNT) bundles can be clearly observed.

The aforementioned CNT-based inductor analyses have assumed perfect contacts (i.e., $R_{\mathrm{mc}}=0$ ), which indicates that maximum performance enhancement (for a given configuration) can be potentially achieved by employing CNT bundles. In reality, the process variability needs to be taken into account. Although some work has achieved nearly perfect contact $\left(R_{\mathrm{mc}} \sim 0\right)$, the value of $R_{\mathrm{mc}}$ could also be as large as tens of kiloohms. Fig. 15 shows the impact of such imperfect contact resistance $\left(R_{\mathrm{mc}}\right)$ on CNT-based inductors. It can be observed that, for the considered range of $R_{\mathrm{mc}}$ per channel, the impact of $R_{\mathrm{mc}}$ is negligible for SWCNT-based inductors but significant for large-diameter MWCNT-based inductors. This is because the number of total conducting channels for SWCNT bundle 

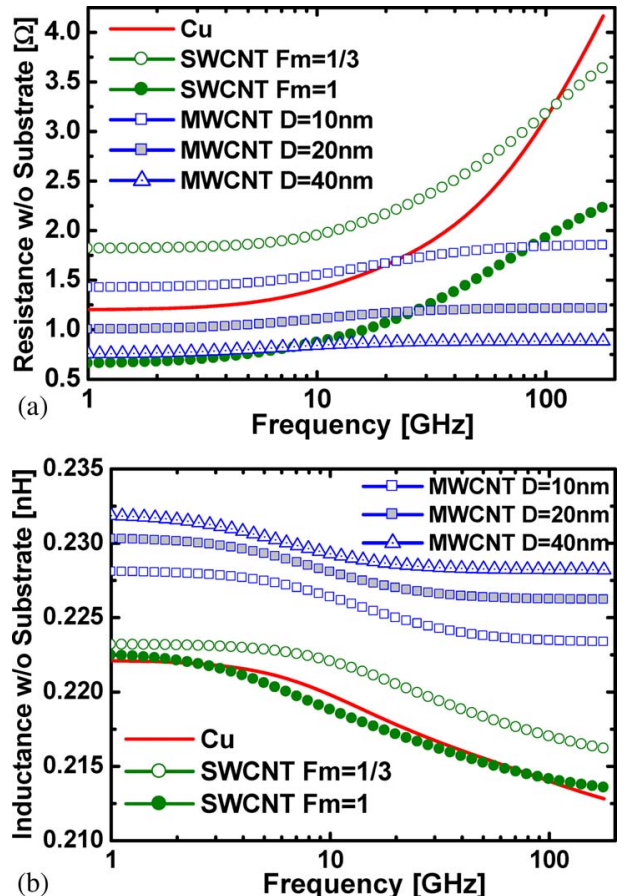

Fig. 14. Total series resistance and inductance $\left(R_{S}\right.$ and $L_{S}$ in Fig. 11) of Cu-, SWCNT-, and MWCNT-based inductors of Fig. 13(a) as a function of frequency.
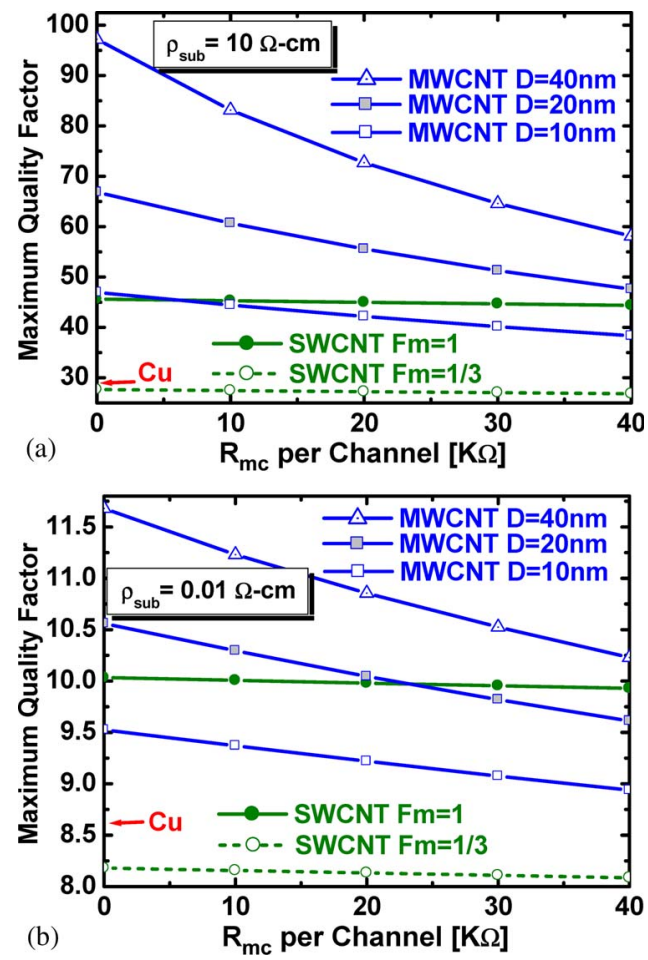

Fig. 15. Maximum $Q$ factor of SWCNT- and MWCNT-based 0.75-turn inductors as a function of imperfect metal-CNT contact resistance per channel $\left(R_{\mathrm{mc}}\right)$, for (a) low-loss substrate $\rho=10 \Omega \cdot \mathrm{cm}$ and (b) high-loss substrate $\rho=0.01 \Omega \cdot \mathrm{cm}$

is very large for a given cross-sectional area (due to small diameter) and the impact of $R_{\mathrm{mc}}$ becomes negligible, whereas for MWCNT bundles, the total number of conducting channels is relatively small due to its large diameter (much fewer nanotubes in a given area) [9] and, thus, $R_{\mathrm{mc}}$ becomes more significant. Aside from $R_{\mathrm{mc}}$, the density of CNTs in the bundle can also be expected to have significant impact on the performance of CNT-based inductors, since the resistance is inversely proportional to the density. It has been shown in [15] that, in order to make CNT inductors outperform $\mathrm{Cu}$ inductors, high CNT density (at least 20\%) will be required.

The preceding analyses are based on the assumption that CNTs are under low-voltage bias, where optical phonon and zone boundary phonon scattering can be excluded. However, at high-voltage bias, these two scattering mechanisms will take effect and increase the resistance of CNTs [24]. For a voltage bias $V$, the total resistance can be written as [24]

$$
R_{\text {total }}=R_{\text {low }}+\frac{V}{I_{\text {sat }}}
$$

where $R_{\text {low }}$ is the resistance used in this work, and $I_{\text {sat }}$ is the saturation current, which is $\sim 25 \mu \mathrm{A}$. From the International Technology Roadmap for Semiconductors [39], the supply voltage of current high-performance RF/analog circuit is $1.2 \mathrm{~V}$ but could be as high as $2.5 \mathrm{~V}$. Considering the case of a 0.75 -turn inductor, even if a voltage of $2.5 \mathrm{~V}$ is assumed, the second term of (38) will only be $5 \%$ of $R_{\text {low }}$. Furthermore, this percentage will be even lower if the total length of the inductor is larger (such as the four-turn inductor discussed in Section IV-B) or if the supply voltage becomes lower. Therefore, it can be concluded that considering low-voltage bias resistance of CNTs is sufficient for on-chip inductor design.

\section{CONCLUSION}

The analysis in this paper has illustrated for the first time that, because of the presence of large kinetic inductance (due to large momentum relaxation time) in each CNT, the skin effect in CNT bundles is significantly reduced compared to that in conventional conductors. Particularly, MWCNTs could offer significant advantages in high-frequency applications, since they exhibit much reduced skin effect in comparison to SWCNTs and are also known to offer comparable circuit performance with that of SWCNTs due to low dc resistivity. This preferable high-frequency property of CNTs is then explored in the design and analysis of high-performance on-chip inductors. It has been demonstrated that the $Q$ factor of CNT-based inductors could be as high as 3.3 times compared to those based on $\mathrm{Cu}$ without using any magnetic materials or any optimization techniques. Since fabricating ideal densely packed SWCNT bundles (100\% metallic nanotubes separated by Van der Waal's gap) remains challenging, large-diameter MWCNT interconnects could be very promising for future high-frequency interconnect applications including inductor design.

\section{APPENDIX I}

For a 1-D conductor with length $L$, and only one conducting channel (two spin modes), the interval of neighboring states in $k$ space is $2 \pi / L$ (shown in Fig. 16). The number of states from zero to $k_{F}$ is equal to $k_{F} /(2 \pi / L)$. Assuming that all available 


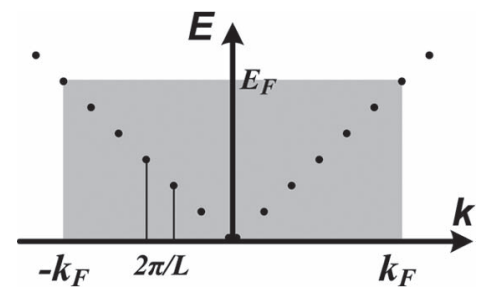

Fig. 16. $E-k$ diagram for a 1-D conductor, where $k_{F}$ is the Fermi wave vector. If the length of $1-\mathrm{D}$ conductor is $L$, the interval of neighboring states in $k$ space is $2 \pi / L$. The shaded area indicates filled states.

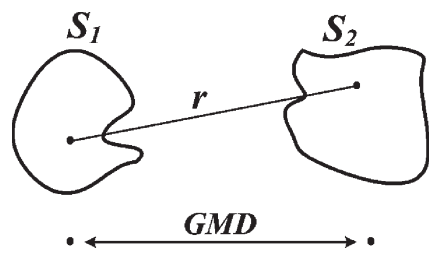

Fig. 17. Illustration of the concept of $G M D$. The mutual inductance between two conductors with arbitrarily shaped cross sections $S_{1}$ and $S_{2}$ can be made equal to the mutual inductance between two simple line conductors, if separated by the $G M D$.

states below $E_{F}$ are filled, the number of carriers per unit length (from $-k_{F}$ to $+k_{F}$ ) can be derived as

$$
n_{1 D}=2 \times 2 \times \frac{k_{F}}{2 \pi}=\frac{2 k_{F}}{\pi}
$$

where $k_{F}$ is the Fermi wave vector. The first prefactor "2" represents the spin degeneracy, and the second prefactor "2" accounts for both negative and positive $k$ space, as shown in Fig. 16.

The conductance of a 1-D conductor is given by

$$
\sigma_{0}^{1 D}=\frac{n_{1 D} e^{2} \tau}{m^{*}}=\frac{2 k_{F} e^{2} \tau}{\pi m^{*}} .
$$

Substituting $\hbar k_{F}=m^{*} \nu$ into (A2), one can obtain

$$
\sigma_{0}^{1 D}=\frac{2 e^{2} \tau \nu}{\pi \hbar}=\frac{4 e^{2} \tau \nu}{h} .
$$

Note that, in a 1-D conductor, the backscattering momentum relaxation time $\tau_{B}=2 \tau$. This is because backscattering corresponds to momentum relaxation from $p$ to $-p$, while normal scattering corresponds to momentum relaxation from $p$ to zero [21]. Therefore, (A3) can be written as

$$
\sigma_{0}^{1 D}=\frac{2 e^{2}}{h} \tau_{B} \nu=\frac{2 e^{2}}{h} \lambda
$$

where the mean free path $\lambda$ is defined as $\lambda=\tau_{B} \nu$.

\section{APPENDIX II}

The concept of geometric mean distance (GMD) is a way of representing the mutual inductance of two conductors with arbitrarily shaped cross sections by two infinitely thin filaments. This concept dates back to 1872 when it was originated by J. C. Maxwell [38]. As shown in Fig. 17, the mutual inductance of two infinitely thin filaments separated by the $G M D$ will be equal to the mutual inductance between two original conductors with arbitrarily shaped cross sections $S_{1}$ and $S_{2}$. The formula used to calculate the GMD for the two conductors with arbitrarily shaped cross sections is given by

$$
\log G M D=\frac{1}{S_{1} S_{2}} \iint \log r d S_{1} d S_{2}
$$

where $r$ is the distance between any pair of points in the cross sections $S_{1}$ and $S_{2}$.

The definition of $G M D$ of a single conductor with arbitrarily shaped cross section is the distance between two infinitely thin imaginary filaments, whose mutual inductance is equal to the self-inductance of the original single conductor.

The arithmetic mean distance (AMD) of two conductors with arbitrarily shaped cross sections is the average distance between every pair of points from one conductor to another. It can be calculated by

$$
A M D=\frac{1}{S_{1} S_{2}} \iint r d S_{1} d S_{2} .
$$

For a single conductor, its $A M D$ is the average distance of all possible pairs of points within the cross section.

\section{APPENDIX III}

The four Maxwell equations can be expressed as

$$
\begin{aligned}
\nabla \times \vec{H} & =\vec{J}+\frac{\partial \vec{D}}{\partial t} \\
\nabla \times \vec{E} & =-\mu \frac{\partial \vec{H}}{\partial t} \\
\nabla \cdot \vec{H} & =0 \\
\nabla \cdot \vec{E} & =\rho
\end{aligned}
$$

where $\vec{H}$ is the magnetic field intensity, $\vec{J}$ is the current density, $\vec{D}$ is the electric displacement field, and $\vec{E}$ is the electric field. Also, Ohm's law can be expressed as

$$
\vec{J}=\sigma \vec{E}
$$

From (A8), one can write the following:

$$
\nabla \times \nabla \times \vec{E}=-\mu \frac{\partial}{\partial t}(\nabla \times \vec{H}) .
$$

Now, recall the vector identity

$$
\nabla \times \nabla \times \vec{E}=\nabla(\nabla \cdot \vec{E})-\nabla^{2} \vec{E} .
$$

Note that there is no source charge in the system $(\rho=0)$, so that (A10) can be written as $\nabla \cdot \vec{E}=0$. Hence, substituting (A13) in (A12), one arrives at

$$
\nabla^{2} \vec{E}=\mu \frac{\partial}{\partial t}(\nabla \times \vec{H}) .
$$

Substituting (A7) and (A11) into (A14) leads to

$$
\nabla^{2} \vec{E}=\mu \frac{\partial}{\partial t}\left(\sigma \vec{E}+\frac{\partial \vec{D}}{\partial t}\right) .
$$

For a good conductor, the displacement current is negligible, compared with the conduction current, so that the second item 
on the right-hand side of (A15) is negligible. Thus, (A15) can be further written as

$$
\nabla^{2} \vec{E}=\mu \sigma \frac{\partial}{\partial t} \vec{E}
$$

For a time-varying field, the electric field can be written as $\vec{E}=\vec{E}_{0} e^{j \omega t}$, so that

$$
\frac{\partial}{\partial t} \vec{E}=\frac{\partial}{\partial t} \vec{E}_{0} e^{j \omega t}=j \omega \vec{E}_{0} e^{j \omega t}=j \omega \vec{E}
$$

Substituting (A17) in (A16), one arrives at

$$
\nabla^{2} \vec{E}=j \omega \mu \sigma \vec{E} .
$$

\section{REFERENCES}

[1] F. Kreupl, A. P. Graham, M. Liebau, G. S. Duesberg, R. Seidel, and E. Unger, "Carbon nanotubes for interconnect applications," in IEDM Tech. Dig., 2004, pp. 683-686.

[2] P. L. McEuen, M. S. Fuhrer, and H. K. Park, "Single-walled carbon nanotube electronics," IEEE Trans. Nanotechnol., vol. 1, no. 1, pp. 7885, Mar. 2002

[3] B. Q. Wei, R. Vajtai, and P. M. Ajayan, "Reliability and current carrying capacity of carbon nanotubes," Appl. Phys. Lett., vol. 79, no. 8, pp. 11721174,2001

[4] H. Li, N. Srivastava, J. F. Mao, W. Y. Yin, and K. Banerjee, "Carbon nanotube vias: A reality check," in IEDM Tech. Dig., 2007, pp. 207-210.

[5] K. Banerjee, H. Li, and N. Srivastava, "Current status and future perspectives of carbon nanotube interconnects," in Proc. 8th Int. Conf. Nanotechnol., 2008, pp. 432-436.

[6] N. Srivastava and K. Banerjee, "Performance analysis of carbon nanotube interconnects for VLSI applications," in Proc. IEEE/ACM ICCAD, 2005, pp. 383-390.

[7] N. Srivastava, H. Li, F. Kreupl, and K. Banerjee, "On the applicability of single-walled carbon nanotubes as VLSI interconnects," IEEE Trans. Nanotechnol., vol. 8, no. 4, pp. 542-559, Jul. 2009.

[8] A. Naeemi and J. D. Meindl, "Design and performance modeling for single-walled carbon nanotubes as local, semiglobal, and global interconnects in gigascale integrated systems," IEEE Trans. Electron Devices, vol. 54, no. 1, pp. 26-37, Jan. 2007

[9] H. Li, W. Y. Yin, K. Banerjee, and J. F. Mao, "Circuit modeling and performance analysis of multi-walled carbon nanotube interconnects," IEEE Trans. Electron Devices, vol. 55, no. 6, pp. 1328-1337, Jun. 2008.

[10] K. Banerjee, S. Im, and N. Srivastava, "Interconnect modeling and analysis in the nanometer era: $\mathrm{Cu}$ and beyond," in Proc. 22nd Adv. Metallization Conf., 2005, pp. 25-31.

[11] W. H. Hayt, Engineering Electromagnetics, 7th ed. New York: McGrawHill, 2006.

[12] P. J. Burke, "Luttinger liquid theory as a model of the gigahertz electrical properties of carbon nanotubes," IEEE Trans. Nanotechnol., vol. 1, no. 3, pp. 129-144, Sep. 2002.

[13] A. Nieuwoudt and Y. Massoud, "Understanding the impact of inductance in carbon nanotube bundles for VLSI interconnect using scalable modeling techniques," IEEE Trans. Nanotechnol., vol. 5, no. 6, pp. 758-765, Nov. 2006.

[14] M. Kamon, M. J. Tsuk, and J. White, "FastHenry: A multipole-accelerated 3-D inductance extraction program," IEEE Trans. Microw. Theory Tech., vol. 42, no. 9, pp. 1750-1758, Sep. 1994.

[15] H. Li and K. Banerjee, "High-frequency effects in carbon nanotube interconnects and implications for on-chip inductor design," in IEDM Tech. Dig., 2008, pp. 525-528.

[16] P. C. P. Watts, W. L. Hsu, D. P. Randall, V. Kotzeva, and G. Z. Chen, "Fe-filled carbon nanotubes: Nano-electromagnetic inductors," Chem. Mater., vol. 14, no. 11, pp. 4505-4508, 2002.

[17] K. Tsubaki, Y. Nakajima, T. Hanajiri, and H. Yamaguchi, "Proposal of carbon nanotube inductors," J. Phys.: Conf. Ser., vol. 38, pp. 49-52, 2006.

[18] A. Nieuwoudt and Y. Massoud, "Predicting the performance of low-loss on-chip inductors realized using carbon nanotube bundles," IEEE Trans. Electron Devices, vol. 55, no. 1, pp. 298-312, Jan. 2008.

[19] N. W. Ashcroft and N. D. Mermin, Solid State Physics. Philadelphia, PA: Saunders College, 1976.

[20] C. Kittel, Introduction to Solid State Physics, 8th ed. Hoboken, NJ: Wiley, 2004.
[21] J. J. Plombon, K. P. O'Brien, F. Gstrein, V. M. Dubin, and Y. Jiao, "High-frequency electrical properties of individual and bundled carbon nanotubes," Appl. Phys. Lett., vol. 90, no. 6, p. 063 106, Feb. 2007.

[22] G. W. Hanson, "Fundamental transmitting properties of carbon nanotube antennas," IEEE Trans. Antennas Propag., vol. 53, no. 11, pp. 3426-3435, Nov. 2005

[23] A. Naeemi and J. D. Meindl, "Compact physical models for multiwall carbon-nanotube interconnects," IEEE Electron Device Lett., vol. 27, no. 5, pp. 338-340, May 2006.

[24] J.-Y. Park, S. Rosenblatt, Y. Yaish, V. Sazonova, H. Ustunel, S. Braig, T. A. Arias, P. W. Brouwer, and P. L. McEuen, "Electron-phonon scattering in metallic single-walled carbon nanotubes," Nano Lett., vol. 4, no. 3 , pp. 517-520, 2004

[25] H. J. Li, W. G. Lu, J. J. Li, X. D. Bai, and C. Z. Gu, "Multichannel ballistic transport in multiwall carbon nanotubes," Phys. Rev. Lett., vol. 95, no. 8, p. 086601 , Aug. 2005.

[26] M. Nihei, T. Hyakushima, S. Sato, T. Nozue, M. Norimatsu, M. Mishima, T. Murakami, D. Kondo, A. Kawabata, M. Ohfuti, and Y. Awano, "Electrical properties of carbon nanotube via interconnects fabricated by novel damascene process," in Proc. Int. Interconnect Technol. Conf., 2007, pp. 204-206.

[27] H. Greenhouse, "Design of planar rectangular microelectronic inductors," IEEE Trans. Parts, Hybrids, Packag., vol. PHP-10, no. 2, pp. 101-109, Jun. 1974.

[28] F. W. Grover, Inductance Calculations. New York: Dover, 2004.

[29] H. Stahl, J. Appenzeller, R. Martel, P. Avouris, and B. Lengeler, "Intertube coupling in ropes of single-wall carbon nanotubes," Phys. Rev. Lett., vol. 85, no. 4, p. 045409, Jul. 2001.

[30] M. Nihei, D. Kondo, A. Kawabata, S. Sato, H. Shioya, M. Sakaue, T. Iwai, M. Ohfuti, and Y. Awano, "Low-resistance multi-walled carbon nanotube vias with parallel channel conduction of inner shells," in Proc. Int. Interconnect Technol. Conf., 2005, pp. 234-236.

[31] A. Kawabata, S. Sato, T. Nozue, T. Hyakushima, M. Norimatsu, M. Mishima, T. Murakami, D. Kondo, K. Asano, M. Ohfuti, H. Kawarada, T. Sakai, M. Nihei, and Y. Awano, "Robustness of CNT via interconnect fabricated by low temperature process over a high-density current," in Proc. IEEE Int. Interconnect Technol. Conf., 2008, pp. 237-239.

[32] K. O, "Estimation methods for quality factor of inductors fabricated in silicon integrated circuit process technologies," IEEE J. Solid-State Circuits, vol. 33, no. 8, pp. 1249-1252, Aug. 1998

[33] P. C. Yue and S. Wong, "Physical modeling of spiral inductors on silicon," IEEE Trans. Electron Devices, vol. 47, no. 3, pp. 560-568, Mar. 2000.

[34] A. Weisshaar and A. Luoh, "Closed-form expressions for the series impedance parameters of on-chip interconnects on multilayer silicon substrates," IEEE Trans. Adv. Packag., vol. 27, no. 1, pp. 126-134, Feb. 2004.

[35] C. Wu, C. Tang, and S. Liu, "Analysis of on-chip spiral inductors using the distributed capacitance model," IEEE J. Solid-State Circuits, vol. 38, no. 6, pp. 1040-1044, Jun. 2003.

[36] X. Guan and A. Hajimiri, "A 24-GHz front-end," IEEE J. Solid-State Circuits, vol. 39, no. 2, pp. 368-373, Feb. 2004.

[37] B. Razavi, "A 60-GHz CMOS receiver front-end," IEEE J. Solid-State Circuits, vol. 41, no. 1, pp. 17-22, Jan. 2006.

[38] T. J. Higgins, "Theory and application of complex logarithms and geometric mean distances," AIEE Trans., vol. 66, pp. 12-16, 1947.

[39] International Technology Roadmap for Semiconductors (ITRS), 2007. [Online]. Available: http://public.itrs.net/

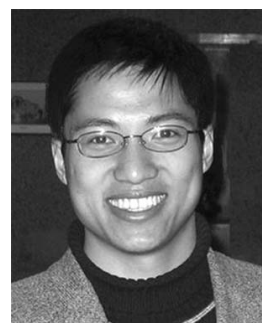

Hong Li (S'07) received the B.E. degree in electronic engineering from Nanjing University of Aeronautics and Astronautics, Nanjing, China, in 2003 and the M.S. degree from the Department of Electronic Engineering, Shanghai Jiao Tong University, Shanghai, China, in 2008. He has been working toward the Ph.D. degree in Professor Banerjee's Nanoelectronics Research Laboratory, Department of Electrical and Computer Engineering, University of California, Santa Barbara (UCSB), since 2007.

Since December 2008, he has been the Lead Graduate Student working on the development of a state-of-the-art carbon nanomaterial fabrication facility at the California NanoSystems Institute, UCSB. His research interests are focused on the modeling and design of carbon nanomaterials for electronic applications-particularly VLSI interconnects and passive devices.

During his undergraduate years, $\mathrm{Mr}$. Li was awarded a number of prizes for outstanding academic performance, including an Excellent Bachelor's Thesis Award. 


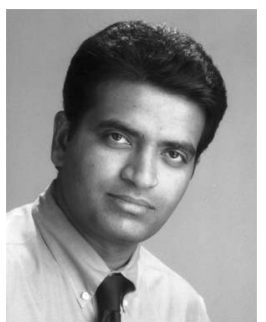

Kaustav Banerjee (S'92-M'99-SM'03) received the $\mathrm{Ph} . \mathrm{D}$. degree in electrical engineering and computer sciences from the University of California, Berkeley, in 1999.

In July 2002, he joined the Faculty of the Department of Electrical and Computer Engineering, University of California, Santa Barbara (UCSB), where he has been a Full Professor since 2007 $\mathrm{He}$ is also an Affiliated Faculty with the California NanoSystems Institute, UCSB. From 1999 to 2001, he was a Research Associate with the Center for Integrated Systems, Stanford University, Stanford, CA. From February to August 2002, he was a Visiting Faculty with the Circuit Research Laboratories, Intel, Hillsboro, OR. He has also held summer/visiting positions at Texas Instruments Incorporated, Dallas, TX, from 1993 to 1997, and the Swiss Federal Institute of Technology, Lausanne, Switzerland, in 2001. His research has been chronicled in over 175 journal and refereed international conference papers and in two book chapters on 3-D ICs. He has also coedited the book Emerging Nanoelectronics: Life With and After CMOS (Springer, 2004). His current research interests include nanometer-scale issues in VLSI as well as circuits and systems issues in emerging nanoelectronics. He is also involved in exploring the design and fabrication of various nanomaterials for ultra energyefficient electronics and energy harvesting/storage applications.

Prof. Banerjee is a Distinguished Lecturer of the IEEE Electron Devices Society. He has served on the Technical Program Committees of several leading IEEE and ACM conferences, including IEDM, DAC, ICCAD, and IRPS. From 2005 to 2008, he served as a member of the Nanotechnology Committee of the IEEE Electron Devices Society. He has also served on the Organizing Committee of ISQED at various positions, including Technical Program Chair in 2002 and General Chair in 2005. He was the recipient of numerous awards in recognition of his work, including a Best Paper Award at the Design Automation Conference in 2001, the ACM SIGDA Outstanding New Faculty Award in 2004, an IEEE Micro Top Picks Award in 2006, and an IBM Faculty Award in 2008 